\title{
A conjecture on equitable vertex arboricity of graphs*
}

\author{
Xin Zhang ${ }^{\mathrm{a} \dagger}$, Jian-Liang $\mathrm{Wu}^{\mathrm{b} \ddagger}$ \\ a Department of Mathematics, Xidian University, Xi' an 710071, P. R. China \\ ${ }^{\mathrm{b}}$ School of Mathematics, Shandong University, Jinan 250100, P. R. China
}

\begin{abstract}
$\mathrm{Wu}$, Zhang and $\mathrm{Li}[4]$ conjectured that the set of vertices of any simple graph $G$ can be equitably partitioned into $\lceil(\Delta(G)+1) / 2\rceil$ subsets so that each of them induces a forest of $G$. In this note, we prove this conjecture for graphs $G$ with $\Delta(G) \geq|G| / 2$.
\end{abstract}

Keywords: Equitable vertex arboricity; Relaxed coloring; Tree coloring; Maximum degree

\section{Introduction}

All graphs considered in this paper are finite, undirected, loopless and without multiple edges. For a graph $G$, we use $V(G), E(G), \delta(G)$ and $\Delta(G)$ to denote the vertex set, the edge set, the minimum degree and the maximum degree of $G$, respectively. By $\alpha^{\prime}(G)$ and $G^{c}$, we denote the largest size of a matching in the graph $G$ and the completement graph of $G$. For other basic undefined concepts we refer the reader to [1].

The vertex-arboricity $a(G)$ of a graph $G$ is the minimum number of subsets into which the vertex set $V(G)$ can be partitioned so that each subset induces a forest. This notation was first introduced by Chartrand, Kronk and Wall [2] in 1968, who named it point-arboricity and proved that $a(G) \leq\lceil(\Delta(G)+1) / 2\rceil$ for every graph $G$. Recently, Wu, Zhang and Li [4] introduced the equitable version of vertex arboricity. If the set of vertices of a graph $G$ can be equitably partitioned into $k$ subsets (i.e. the size of each subset is either $\lceil|G| / k\rceil$ or $\lfloor|G| / k\rfloor$ ) such that each subset of vertices induce a forest of $G$, then we call that $G$ admits an equitable $k$-tree-coloring. The minimum integer $k$ such that $G$ has an equitable $k$-tree-coloring is the equitable vertex arboricity $a_{e q}(G)$ of $G$. As an extension of the result of Chartrand, Kronk and Wall on vertex arboricity, Wu, Zhang and

\footnotetext{
*This research is supported by the National Natural Science Foundation of China (No. 11101243, 11201440).

$\dagger$ Email address: xzhang@xidian.edu.cn

†Email address: jlwu@sdu.edu.cn
} 
Li [4] raised the following conjecture and they proved it for complete bipartite graphs, graphs with maximum average degree less than 3 , and graphs with maximum average degree less than 10/3 and maximum degree at least 4.

Conjecture 1.1. $a_{e q}(G) \leq\left\lceil\frac{\Delta(G)+1}{2}\right\rceil$ for every simple graph $G$.

In this note, we establish this conjecture for graphs $G$ with $\Delta(G) \geq|G| / 2$.

\section{Main results and the proofs}

For convenience, we set $\Gamma(G)=\left\lceil\frac{\Delta(G)+1}{2}\right\rceil$ throughout this section. To begin with, we introduce two useful lemmas of Chen, Lih and $\mathrm{Wu}$.

Lemma 2.1. [3] If $G$ is a disconnected graph, then $\alpha^{\prime}(G) \geq \delta(G)$.

Lemma 2.2. [3] If $G$ is a connected graph such that $|G|>2 \delta(G)$, then $\alpha^{\prime}(G) \geq \delta(G)$.

Lemma 2.3. If $G$ is a connected graph with $\delta(G) \geq 2$, then $G$ contains a cycle of length at least $\delta(G)+1$.

Proof. Consider the longest path $P=\left[v_{0} v_{1} \ldots v_{k}\right]$ in $G$. We see immediately that $N\left(v_{0}\right) \subseteq V(P)$, because otherwise we would construct a longer path. Let $v_{i}$ be a neighbor of $v_{0}$ so that $i$ is maximum. Since $\delta(G) \geq 2, C=\left[v_{0} v_{1} \ldots v_{i} v_{0}\right]$ is a cycle of length $i+1 \geq \delta(G)+1$.

In what follows, we prove three independent theorems, which together imply Conjecture 1.1 for graphs $G$ with $\Delta(G) \geq|G| / 2$.

Theorem 2.4. If $\Delta(G) \geq \frac{2}{3}|G|-1$, then $a_{e q}(G) \leq \Gamma(G)$.

Proof. If $\Delta(G)=|G|-1$, then $a_{e q}(G) \leq \Gamma(G)$ and this upper bound can be attained by the complete graphs, since we can arbitrarily partition $V(G)$ into $\Gamma(G)$ subsets so that each of them consists of one or two vertices, thus we assume $\Delta(G) \leq|G|-2$. Since $\Delta(G)+\delta\left(G^{c}\right)=|G|-1$ and $\Delta(G) \geq \frac{2}{3}|G|-1$, $\left|G^{c}\right| \geq 3 \delta\left(G^{c}\right)$ and $\delta\left(G^{c}\right) \geq\left|G^{c}\right|-2 \Gamma(G)$. By Lemmas 2.1 and 2.2, we have $\alpha^{\prime}\left(G^{c}\right) \geq \delta\left(G^{c}\right)$, so there exists a matching $M=\left[x_{1} y_{1}, \ldots, x_{\delta} y_{\delta}\right]$ of size $\delta:=\delta\left(G^{c}\right)$ in $G^{c}$. Since $\left|G^{c}\right| \geq 3 \delta\left(G^{c}\right)$, $\left|V\left(G^{c}\right) \backslash V(M)\right| \geq \delta$, thus we can select $\delta$ distinct vertices $z_{1}, \ldots, z_{\delta}$ among $V\left(G^{c}\right) \backslash V(M)$. Denote $\beta=\left|G^{c}\right|-2 \Gamma(G)$ and $\mu=3 \Gamma(G)-\left|G^{c}\right|$. Since $|G|-2 \geq \Delta(G) \geq \frac{2}{3}|G|-1, \beta, \mu \geq 0$. We now use $\beta$ colors to color $3 \beta$ vertices of $G$ so that the $i$-th color class consists of the three vertices $x_{i}, y_{i}$ and $z_{i}$, and then use $\mu$ colors to color the remaining $2 \mu$ vertices of $G$ so that each color class consists of two vertices. One can check that each color class of $G$ induces a (linear) forest and the coloring of $G$ is equitable. Therefore, $a_{e q}(G) \leq \beta+\mu=\Gamma(G)$. 
Theorem 2.5. If $\frac{2}{3}|G|-1>\Delta(G) \geq \frac{2}{3}|G|-2$, then $a_{e q}(G) \leq \Gamma(G)$.

Proof. If $|G| \leq 3$, then the result is trivial, so we assume $|G| \geq 4$. If $|G|=3 k$, then $\Delta(G)=2 k-2$ and $\delta\left(G^{c}\right)=k+1$, since $\frac{2}{3}|G|-1>\Delta(G) \geq \frac{2}{3}|G|-2$ and $\Delta(G)+\delta\left(G^{c}\right)=|G|-1$. By Lemmas 2.1 and 2.2, we have $\alpha^{\prime}\left(G^{c}\right) \geq \delta\left(G^{c}\right)>k$. Let $M_{1}=\left[x_{11} y_{11}, \ldots, x_{1 k} y_{1 k}\right]$ be a matching of $G^{c}$. We now partition the vertices of $G$ into $k$ subsets so that the $i$-th subset consists of the vertices $x_{1 i}, y_{1 i}$ and one another vertex different from the vertices in $V\left(M_{1}\right)$. It is easy to check that this is an equitable partition so that each subset induces a (linear) forest, therefore, $a_{e q}(G) \leq k=\Gamma(G)$. If $|G|=3 k+2$, then $\Delta(G)=2 k$ and $\delta\left(G^{c}\right)=k+1$. This also implies, by Lemma 2.1 and 2.2 , that $\alpha^{\prime}\left(G^{c}\right) \geq \delta\left(G^{c}\right)>k$. Let $M_{2}=\left[x_{21} y_{21}, \ldots, x_{2 k} y_{2 k}\right]$ be a matching of $G^{c}$. We now partition the vertices of $G$ into $k+1$ subsets so that the $i$-th subset with $i \leq k$ consists of the vertices $x_{2 i}, y_{2 i}$ and one another vertex different from the vertices in $V\left(M_{2}\right)$ and the $(k+1)$-th subset consists of two vertices in $V(G) \backslash V\left(M_{2}\right)$. It is easy to check that this is an equitable partition so that each subset induces a (linear) forest, therefore, $a_{e q}(G) \leq k+1=\Gamma(G)$. If $|G|=3 k+1$, then $\Delta(G)=2 k-1$ and $\delta\left(G^{c}\right)=k+1$. By Lemmas 2.1 and 2.2, we have $\alpha^{\prime}\left(G^{c}\right) \geq \delta\left(G^{c}\right)$. Let $M_{3}=\left[x_{31} y_{31}, \ldots, x_{3(k+1)} y_{3(k+1)}\right]$ be a matching of $G^{c}$. If $x_{31}$ has a neighbor in $G^{c}$ among $\left\{x_{32}, y_{32}, \ldots, x_{3(k+1)}, y_{3(k+1)}\right\}$ (without loss of generality, assume that $x_{31} x_{32} \in E\left(G^{c}\right)$ ), then we can partition the vertices of $G$ into $k$ subsets so that the the first subset consists of the four vertices $x_{31}, y_{31}, x_{32}$ and $y_{32}$ and the $i$-th subset with $2 \leq i \leq k$ consists of the vertices $x_{3(i+1)}, y_{3(i+1)}$ and one another vertex different from the vertices in $V\left(M_{2}\right)$. One can check that this is an equitable partition so that each subset induces a (linear) forest, therefore, $a_{e q}(G) \leq k=\Gamma(G)$. Hence, we shall assume that $x_{31} x_{3 j}, x_{31} y_{3 j} \notin E\left(G^{c}\right)$ for each $2 \leq j \leq k+1$. Since $d_{G^{c}}\left(x_{31}\right) \geq \delta\left(G^{c}\right)=k+1$ and $\left|G^{c}\right|=3 k+1, x_{31} z \in E\left(G^{c}\right)$ for each $z \in V\left(G^{c}\right) \backslash V\left(M_{3}\right)$. Similarly, we shall assume that $y_{31} z \in E\left(G^{c}\right)$ for each $z \in V\left(G^{c}\right) \backslash V\left(M_{3}\right)$, because otherwise we return to a case we have considered before. We now partition the vertices of $G$ into $k$ subsets so that the the first subset consists of the two vertices $x_{31}, y_{31}$ and two distinct vertices $z_{1}, z_{2} \in V\left(G^{c}\right) \backslash V\left(M_{3}\right)$ and the $i$-th subset with $2 \leq i \leq k$ consists of the vertices $x_{3 i}, y_{3 i}$ and one another vertex different from the vertices in $V\left(M_{2}\right)$. One can again check that this is an equitable partition so that each subset induces a (linear) forest, therefore, $a_{e q}(G) \leq k=\Gamma(G)$.

Theorem 2.6. If $\frac{2}{3}|G|-2>\Delta(G) \geq \frac{1}{2}|G|$, then $a_{e q}(G) \leq \Gamma(G)$.

Proof. Since $\Delta(G)+\delta\left(G^{c}\right)=|G|-1$ and $\Delta(G) \geq \frac{1}{2}|G|,\left|G^{c}\right| \geq 2 \delta\left(G^{c}\right)+2$. We split our proof into two cases.

Case 1: $G^{c}$ is connected.

Since $\left|G^{c}\right| \geq 2 \delta\left(G^{c}\right)+2>2 \delta\left(G^{c}\right)$, there exists a path $P=\left[x_{0}, x_{1}, \ldots, x_{2 \delta}\right]$ of length $2 \delta:=2 \delta\left(G^{c}\right)$ in $G^{c}$ (see [1, Exercise 4.2.9]). Denote $\beta=|G|-3 \Gamma(G)$ and $\mu=4 \Gamma(G)-|G|$. Since $\frac{2}{3}|G|-2>$ $\Delta(G) \geq \frac{1}{2}|G|, \beta, \mu \geq 1$. Since $2 \Gamma(G)>\Delta(G)=|G|-\delta\left(G^{c}\right)-1, \delta\left(G^{c}\right) \geq|G|-2 \Gamma(G)=2 \beta+\mu$. 
Thus, the vertex sets $V_{i}=\left\{x_{4 i-4}, x_{4 i-3}, x_{4 i-2}, x_{4 i-1}\right\}$ with $1 \leq i \leq \beta$ and $U_{i}=\left\{x_{4 \beta+2 i-2}, x_{4 \beta+2 i-1}\right\}$ with $1 \leq i \leq \mu$ are well defined. Note that $V(P) \supseteq \bigcup_{i=1}^{\beta} V_{i} \cup \bigcup_{i=1}^{\mu} U_{i}$. Since $|G|-4 \beta-3 \mu=\mu$, $\left|G-\bigcup_{i=1}^{\beta} V_{i}-\bigcup_{i=1}^{\mu} U_{i}\right|=\mu$. Let $V(G) \backslash\left(\bigcup_{i=1}^{\beta} V_{i} \cup \bigcup_{i=1}^{\mu} U_{i}\right)=\left\{y_{1}, \ldots, y_{\mu}\right\}$ and let $W_{i}=U_{i} \cup\left\{y_{i}\right\}$ with $1 \leq i \leq \mu$. We now partition the vertices of $G$ into $\beta+\mu$ subsets $V_{1}, \ldots, V_{\beta}, W_{1}, \ldots, W_{\mu}$. One can check that this is an equitable partition so that each subset induces a (linear) forest, therefore, $a_{e q}(G) \leq \beta+\mu=\Gamma(G)$.

Case 2: $G^{c}$ is disconnected.

Let $G_{1}, \ldots, G_{t}$ be the components of $G^{c}$ with $t \geq 2$. Since $\Delta(G)+\delta\left(G^{c}\right)=|G|-1$ and $\Delta(G)<\frac{2}{3}|G|-2, \min \left\{\delta\left(G_{1}\right), \ldots \delta\left(G_{t}\right)\right\} \geq \delta\left(G^{c}\right) \geq 2$. This implies, by Lemma 2.3, that $G_{i}$ contains a cycle $C_{i}=\left[x_{0}^{i} x_{1}^{i} \ldots x_{l\left(C_{i}\right)}^{i} x_{0}^{i}\right]$ of length $l\left(C_{i}\right)+1 \geq \delta\left(G_{i}\right)+1$ for each $1 \leq i \leq t$. Let $V_{j}^{i}=\left\{x_{4 j-4}^{i}, x_{4 j-3}^{i}, x_{4 j-2}^{i}, x_{4 j-1}^{i}\right\}$ with $1 \leq i \leq t$ and $1 \leq j \leq n_{i}$, in which $4 n_{i}-1 \leq l\left(C_{i}\right)$ and $n_{1}+\ldots+n_{t}=\beta$. Note that $V_{j}^{i}$ is well defined by Claim 1 .

Claim 1. $\sum_{i=1}^{t}\left\lfloor\frac{\delta\left(G_{i}\right)+1-4 n_{i}}{2}\right\rfloor \geq \mu$.

Proof. Otherwise, $\delta\left(G^{c}\right) \leq \frac{1}{2} t \delta\left(G^{c}\right) \leq \frac{1}{2} \Sigma_{i=1}^{t} \delta\left(G_{i}\right) \leq \Sigma_{i=1}^{t}\left\lfloor\frac{\delta\left(G_{i}\right)+1}{2}\right\rfloor<2 \beta+\mu=|G|-2 \Gamma(G) \leq$ $|G|-\Delta(G)-1$, contradicting to $\Delta(G)+\delta\left(G^{c}\right)=|G|-1$.

We conclude, by Claim 1 , that there exists a matching $M$ of size at least $\mu$ in $G^{c}-\bigcup_{i=1}^{t} \bigcup_{j=1}^{n_{i}} V_{j}^{i}$. Therefore, we can partition the vertices of $G$ into $\beta+\mu$ subsets so that the $i$-th subset with $1 \leq i \leq \mu$ consists of a pair of vertices matched under $M$ and one vertex in $V(G) \backslash\left(V(M) \cup \bigcup_{i=1}^{t} \bigcup_{j=1}^{n_{i}} V_{j}^{i}\right)$ and the last $\beta$ subsets are $V_{1}^{1}, \ldots, V_{n_{1}}^{1}, \ldots, V_{1}^{t}, \ldots, V_{n_{t}}^{t}$. One can check that this is an equitable partition so that each subset induces a (linear) forest, therefore, $a_{e q}(G) \leq \beta+\mu=\Gamma(G)$.

From the proofs of the above three theorems, we can immediately deduce the following conclusions.

Conclusion 2.7. If $G$ is a simple graph with $\Delta(G) \geq \frac{1}{2}|G|$, then $|V(G)|$ can be equitably partitioned into $\Gamma(G)$ subsets so that each of them induces a linear forest of $G$, i.e., the equitable linear vertex arboricity of $G$ is at most $\Gamma(G)$, and the upper bound $\Gamma(G)$ is sharp.

Conclusion 2.8. An equitable $\Gamma(G)$-tree-coloring of any simple graph $G$ can be constructed in linear time.

\section{References}

[1] J. A. Bondy, U. S. R. Murty, Graph Theory with Applications, North-Holland, New York, 1976. 
[2] G. Chartrand, H. V. Kronk, C. E. Wall, The point-arboricity of a graph, Israel J. Math., 6 (1968), 169-175.

[3] B.-L. Chen, K.-W. Lih, P.-L. Wu, Equitable coloring and the maximum degree, Europ. $L$. Combinatorics, 15 (1994), 443-447.

[4] J.-L. Wu, X. Zhang, H. Li, Equitable vertex arboricity of graphs, Discrete Mathematics, submitted for publication. 